\section{Comments on the Use of Digital Filters}

\author{
Scott D. Walck \\ PPG Industries, Inc. \\ SWalck@dashfast.com
}

At the Microscopy and Microanalysis 2003 meeting, a member of the audience in one talk sharply criticized a speaker of a talk for his use of a Savitsky-Golay (S-G) digital filter ${ }^{1,2,3}$ for smoothing Electron Energy Loss Spectroscopy (EELS) data. The statement was made that the digital filter would decrease the resolution of the peak, i.e. widen the width, and that all digital filters do that, including the Top Hat filtert. In support of this statement, he said to consider what would happen if the filter were applied to a Dirac delta function. I knew that his argument was not correct, but I didn't think of the reason until later. This interchange was on my mind because I was familiar with both of these filters due to implementing both the Top Hat and the Savitsky-Golay filters into my "Beerware " program, EELS-PLOT. ${ }^{3}$ It is well known that the width of the Top Hat filter should be approximately the width of the peaks that it is operating on. After re-examining Madden's paper, I also found the statement that the optimum size of the S-G filter is dependent on its application, i.e. that its optimal width is dependent on the width of the spectral peaks that it is applied to. Recently, I had the need to apply the filter to an X-ray Photoelectron Spectroscopy (XPS) peak that suffered from a poor signal to noise ratio since the concentration was low. The Savitsky-Golay filter was designed for this purpose, and this signal was ideal to examine the effects of increasing the size of the S-G filter. Those results will be presented below, together with my analysis.

The S-G digital filter operates on spectral data in the same way as the Top Hat filter operates, by sliding an array of coefficients through the spectrum in which, at each point, the value of the point is replaced by the sum of the coefficients multiplied by the corresponding nearest neighbors in the original spectral array. In the case of the smoothing S-G filter, the coefficients are those that would fit an $n^{\text {th }}$ degree polynomial. At each point of the spectral array, there are $m$ coefficients on either side of the center value. This leads to the $S-G$ filter being $2 m+1$ in size. For the $1^{\text {st }}$ and $2^{\text {nd }} \mathrm{S}-\mathrm{G}$ derivatives, the coefficients are those corresponding to the fitted $1^{\text {st }}$ and $2^{\text {nd }}$ digital derivatives. The coefficients for the smoothing and first two derivatives of the S-G filters for a quadratic polynomial, $n=2$, are given by the following expressions:

$$
\text { smoothing, for } s=-m \text { to } m, C_{S}=\frac{3\left(3 m^{2}+3 m-1-5 s^{2}\right)}{(2 m+3)(2 m+1)(2 m-1)}
$$

$$
1 \text { st deriv., for } s=-m \text { to } m, C_{S}^{\prime}=\frac{3 s}{(2 m+1)(m+1)(m)}
$$

$$
2 n d \text { deriv., for } s=-m \text { to } m, C_{s}=\frac{30\left\{3 s^{2}-m(m+1)\right\}}{(2 m+3)(2 m+1)(2 m-1)(m+1)(m)}
$$

Tables 1-3 give the actual coefficients used for the quadratic fit for values of $m$ up to 12. It is interesting to note the symmetry of the coefficients and that the smoothing coefficients sum to one. For an odd-ordered derivative they are anti-symmetric and for an even-ordered derivative they are symmetric, while for all them, they sum to zero. Let's see what these filters do to a typical XEDS spectrum. Figure 1 shows the Ni- $K$ peaks results from the application of a 7-pt and 25-pt S-G filter applied to an XEDS

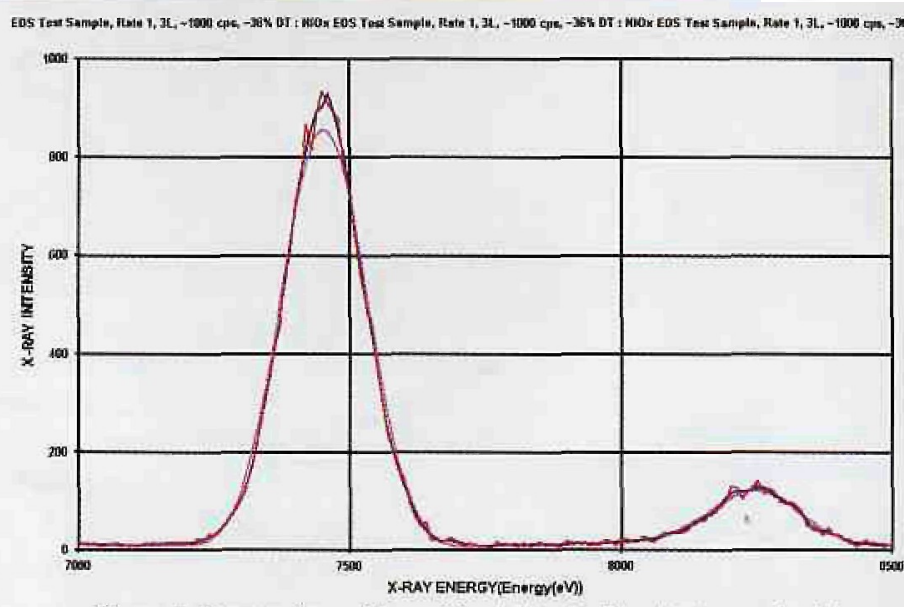

Figure 1 Comparison of the original data (red), a 7-pt smoothed data (blue), and a 25-pt smoothed data (magenta) for an XEDS peak from a TEM test sample is given below.

spectrum from a NiOX ${ }^{\prime \prime}$ analytical TEM test specimen in the EELS-Plot program. The number of channels for the FWHM for the Ni-Ka peak is about 16 . Figure 2 shows the comparison of the 7-pt, 15-pt, and 25-pt S-G $1^{\text {st }}$ derivatives applied to the same spectrum. Figure 3 shows the 7-pt, $15-\mathrm{pt}$, and 25 -pt $2^{\text {nd }}$ derivatives and for fun, I've included the results of a Top Hat Filter applied to the spectrum for comparison. The width of the Top Hat filter is 32 channels with the first and last 8 channel values equal to -1 and the middle 16 values equal to +1 .

The results show that if the width of the S-G filter is chosen to agree with the width of the peak, then it closely matches the peak. However, if the width of the S-G filter is much greater, then it cannot follow the curvature of a peak that has a high signal to noise ratio. Ideally, it should be less than the width in order to follow the curvature of the peak, but if it is too small, then it starts to follow the fluctuations in the data. Examination of the curves for the $\mathrm{Ni}-K \beta$ in Figure 1 shows that for peaks with a smaller signal to noise ratio, the $S-G$ is a good fit even for filters that have a higher number of points. The basic difference is that the higher signal has higher curvature at the maximum value and the quadratic

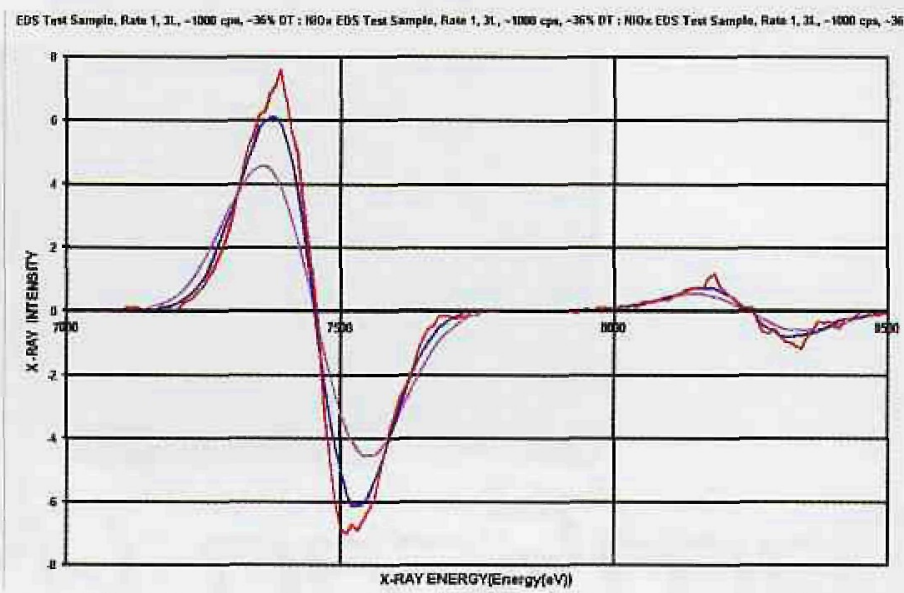

Figure 2 A comparison between a 7-pt (red), 15-pt (biue), and 25-pt (magenta) 1st derivatives for the same peaks in Figure 1.

"BeerWare" is a close cousin to "FreeWare" with a unique microscopy flavor. If you encounter the author at a conference, particularly a Microscopy and Microanalysis meeting, you owe him a glass of beer and your comments about the program. If you find it useful, you owe him a pitcher of beer to share with you and you must extol his virtues to the EM gods. 


\section{Powering Discovery}

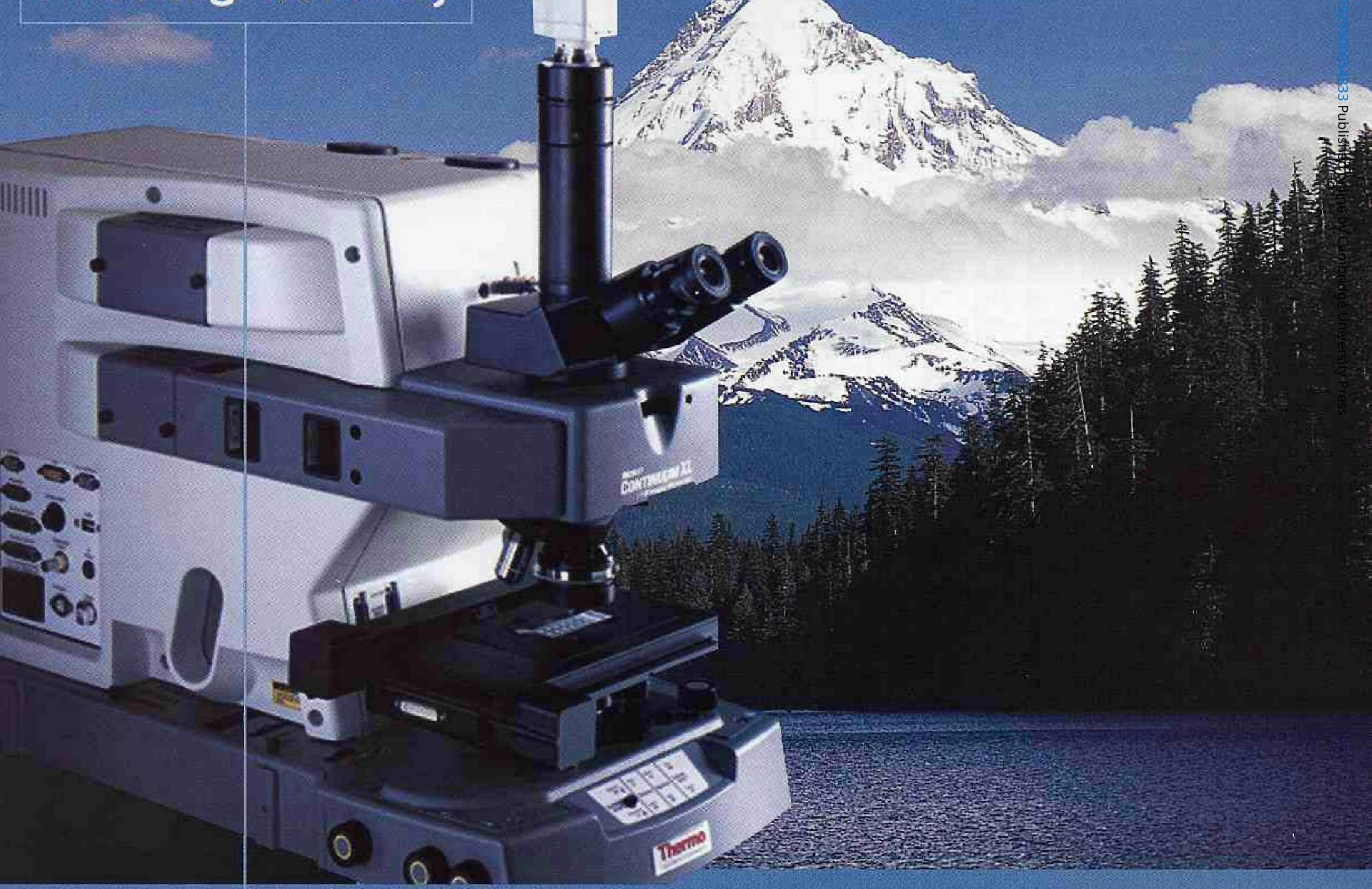

The New Nicolet

Continuum $\mathrm{m}^{\mathrm{TM}} \mathrm{XL}$

\section{Superior Imaging}

Spatial Resolution

\section{Greater Clarity}

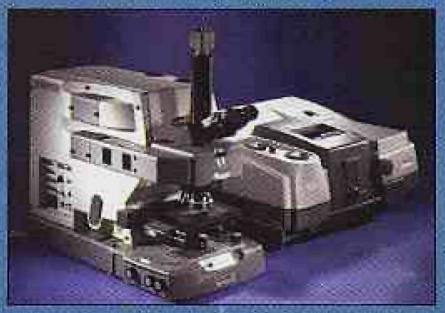

Thermo Electron introduces the Nicolet Continupum XL, a research-quality FFIR microscope with the most advanced imaging available.

Unlock even your most mystifying sample, The innovative imaging design provides full spechal range with unprecedented image quality.

Improve your throughput. A dual-masking remote aperture provides a continuous view of the sample while simultaneously collecting the purest spectral data.

Soar beyond current limitations. Couple the revolutionary imaging of this instrument with the power of Nicolet ${ }^{m}$ FT-IR spectrometers.

See the future of imaging today. Contact Therme Electron at 1-800-532-4752, email analyze@thermo:conn or visit Www:themo.com/spectroscopy 
Table 1: Savitsky-Golay Smoothing Coefficients for Quadratic Fit

\begin{tabular}{|c|r|r|r|r|r|r|r|r|r|r|r|r|}
\hline & $m$ & 2 & 3 & 4 & 5 & 6 & 7 & 8 & 9 & 10 & 11 & 12 \\
\hline 5 & $2 m+1$ & 5 & 7 & 9 & 11 & 13 & 15 & 17 & 19 & 21 & 23 & 25 \\
\hline & & & & & & & & & & & & \\
\hline-12 & & & & & & & & & & & & -0.0489 \\
\hline-11 & & & & & & & & & & & -0.0522 & -0.0267 \\
\hline-10 & & & & & & & & & & -0.0559 & -0.0261 & -0.0064 \\
\hline-9 & & & & & & & & & -0.0602 & -0.0248 & -0.0025 & 0.0120 \\
\hline-8 & & & & & & & & -0.0650 & -0.0226 & 0.0029 & 0.0186 & 0.0284 \\
\hline-7 & & & & & & & -0.0706 & -0.0186 & 0.0106 & 0.0275 & 0.0373 & 0.0429 \\
\hline-6 & & & & & & -0.0769 & -0.0118 & 0.0217 & 0.0394 & 0.0487 & 0.0534 & 0.0555 \\
\hline-5 & & & & & -0.0839 & 0.0000 & 0.0380 & 0.0557 & 0.0637 & 0.0667 & 0.0671 & 0.0661 \\
\hline-4 & & & & -0.0909 & 0.0210 & 0.0629 & 0.0787 & 0.0836 & 0.0836 & 0.0814 & 0.0783 & 0.0748 \\
\hline-3 & & & -0.0952 & 0.0606 & 0.1026 & 0.1119 & 0.1104 & 0.1053 & 0.0991 & 0.0928 & 0.0870 & 0.0815 \\
\hline-2 & & -0.0857 & 0.1429 & 0.1688 & 0.1608 & 0.1469 & 0.1330 & 0.1207 & 0.1101 & 0.1010 & 0.0932 & 0.0864 \\
\hline-1 & & 0.3429 & 0.2857 & 0.2338 & 0.1958 & 0.1678 & 0.1466 & 0.1300 & 0.1168 & 0.1059 & 0.0969 & 0.0893 \\
\hline 0 & & 0.4857 & 0.3333 & 0.2554 & 0.2075 & 0.1748 & 0.1511 & 0.1331 & 0.1190 & 0.1076 & 0.0981 & 0.0902 \\
\hline 1 & & 0.3429 & 0.2857 & 0.2338 & 0.1958 & 0.1678 & 0.1466 & 0.1300 & 0.1168 & 0.1059 & 0.0969 & 0.0893 \\
\hline 2 & & -0.0857 & 0.1429 & 0.1688 & 0.1608 & 0.1469 & 0.1330 & 0.1207 & 0.1101 & 0.1010 & 0.0932 & 0.0864 \\
\hline 3 & & & -0.0952 & 0.0606 & 0.1026 & 0.1119 & 0.1104 & 0.1053 & 0.0991 & 0.0928 & 0.0870 & 0.0815 \\
\hline 4 & & & & -0.0909 & 0.0210 & 0.0629 & 0.0787 & 0.0836 & 0.0836 & 0.0814 & 0.0783 & 0.0748 \\
\hline 5 & & & & & -0.0839 & 0.0000 & 0.0380 & 0.0557 & 0.0637 & 0.0667 & 0.0671 & 0.0661 \\
\hline 6 & & & & & & -0.0769 & -0.0118 & 0.0217 & 0.0394 & 0.0487 & 0.0534 & 0.0555 \\
\hline 7 & & & & & & & -0.0706 & -0.0186 & 0.0106 & 0.0275 & 0.0373 & 0.0429 \\
\hline 8 & & & & & & & & -0.0650 & -0.0226 & 0.0029 & 0.0186 & 0.0284 \\
\hline 9 & & & & & & & & & -0.0602 & -0.0248 & -0.0025 & 0.0120 \\
\hline 10 & & & & & & & & & & -0.0559 & -0.0261 & -0.0064 \\
\hline 11 & & & & & & & & & & & -0.0522 & -0.0267 \\
\hline 12 & & & & & & & & & & & & -0.0489 \\
\hline
\end{tabular}

Table 2: Savitsky-Golay First Derivative Coefficients for Quadratic Fit

\begin{tabular}{|r|r|r|r|r|r|r|r|r|r|r|r|r|}
\hline & $\mathrm{m}$ & 2 & 3 & 4 & 5 & 6 & 7 & 8 & 9 & 10 & 11 & 12 \\
\hline 5 & $2 \mathrm{~m}+1$ & 5 & 7 & 9 & 11 & 13 & 15 & 17 & 19 & 21 & 23 & 25 \\
\hline & & & & & & & & & & & & \\
\hline-12 & & & & & & & & & & & -0.00923 \\
\hline-11 & & & & & & & & & & & -0.01087 & -0.00846 \\
\hline-10 & & & & & & & & & & -0.01299 & -0.00988 & -0.00769 \\
\hline-9 & & & & & & & & & -0.01579 & -0.01169 & -0.00889 & -0.00692 \\
\hline-8 & & & & & & & & -0.01961 & -0.01404 & -0.01039 & -0.00791 & -0.00615 \\
\hline-7 & & & & & & & -0.02500 & -0.01716 & -0.01228 & -0.00909 & -0.00692 & -0.00538 \\
\hline-6 & & & & & & -0.03297 & -0.02143 & -0.01471 & -0.01053 & -0.00779 & -0.00593 & -0.00462 \\
\hline-5 & & & & & -0.04545 & -0.02747 & -0.01786 & -0.01225 & -0.00877 & -0.00649 & -0.00494 & -0.00385 \\
\hline-4 & & & & -0.06667 & -0.03636 & -0.02198 & -0.01429 & -0.00980 & -0.00702 & -0.00519 & -0.00395 & -0.00308 \\
\hline-3 & & & -0.10714 & -0.05000 & -0.02727 & -0.01648 & -0.01071 & -0.00735 & -0.00526 & -0.00390 & -0.00296 & -0.00231 \\
\hline-2 & & -0.200 & -0.07143 & -0.03333 & -0.01818 & -0.01099 & -0.00714 & -0.00490 & -0.00351 & -0.00260 & -0.00198 & -0.00154 \\
\hline-1 & & -0.100 & -0.03571 & -0.01667 & -0.00909 & -0.00549 & -0.00357 & -0.00245 & -0.00175 & -0.00130 & -0.00099 & -0.00077 \\
\hline 0 & & 0.0 & 0.0 & 0.0 & 0.0 & 0.0 & 0.0 & 0.0 & 0.0 & 0.0 & 0.0 & 0.0 \\
\hline 1 & & 0.100 & 0.03571 & 0.01667 & 0.00909 & 0.00549 & 0.00357 & 0.00245 & 0.00175 & 0.00130 & 0.00099 & 0.00077 \\
\hline 2 & & 0.200 & 0.07143 & 0.03333 & 0.01818 & 0.01099 & 0.00714 & 0.00490 & 0.00351 & 0.00260 & 0.00199 & 0.00154 \\
\hline 3 & & & 0.10714 & 0.05000 & 0.02727 & 0.01648 & 0.01071 & 0.00735 & 0.00526 & 0.00390 & 0.00296 & 0.00231 \\
\hline 4 & & & & 0.06667 & 0.03636 & 0.02196 & 0.01429 & 0.00980 & 0.00702 & 0.00519 & 0.00395 & 0.00308 \\
\hline 5 & & & & & 0.04545 & 0.02747 & 0.01786 & 0.01225 & 0.00877 & 0.00649 & 0.00494 & 0.00385 \\
\hline 6 & & & & & 0.03297 & 0.02143 & 0.01471 & 0.01053 & 0.00779 & 0.00593 & 0.00462 \\
\hline 7 & & & & & & & 0.02500 & 0.01716 & 0.01228 & 0.00909 & 0.00692 & 0.00538 \\
\hline 6 & & & & & & & & 0.01961 & 0.01404 & 0.01039 & 0.00791 & 0.00615 \\
\hline 9 & & & & & & & & & 0.01579 & 0.01169 & 0.00889 & 0.00692 \\
\hline 10 & & & & & & & & & & 0.01299 & 0.00989 & 0.00769 \\
\hline 11 & & & & & & & & & & & 0.01087 & 0.00846 \\
\hline 12 & & & & & & & & & & & & 0.00923 \\
\hline
\end{tabular}

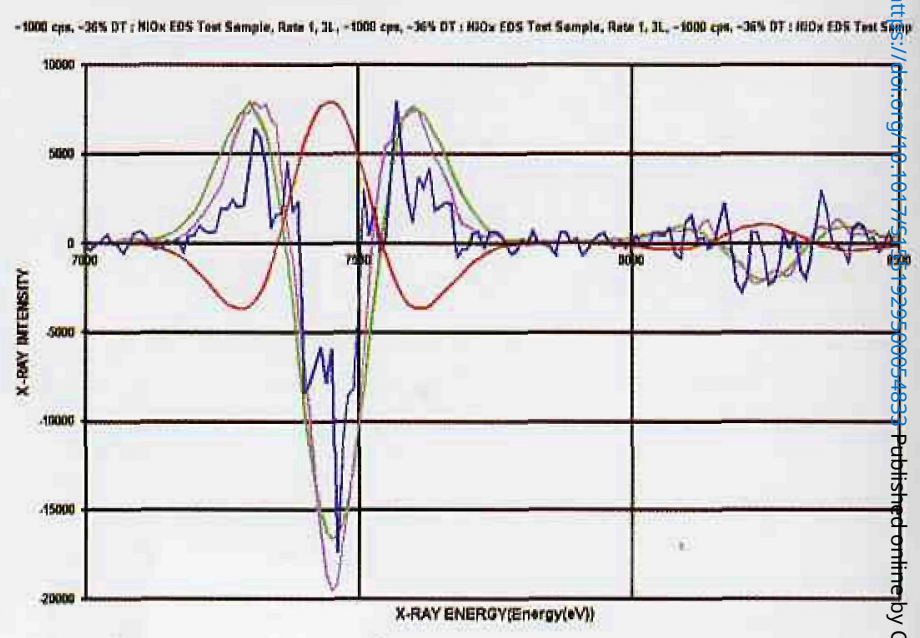

Figure 3 A comparison between a 32-pt $(8,16,8)$ Top Hat filter (red), 7-pt (blue), 15-pt (magenta), and 25-pt (green) 2nd derivatives for the same

peaks in Figure 1. The curves are scaled to the same height. expression cannot match it because of the constraining points of the sides of the peak. An example of a very weak XPS peak wigh be used to examine the $S-G$ curve applied to a small noisy signat. Figure 4 shows a $\mathrm{N}$ 1s peak that is the result of the summation of 100 scans with long dwell times and is close to the sensitivity limit for the instrument. The continuous curve fitting the actual spectral points is the result of the smoothing option chosen in the Microsoft ${ }^{*}$ Excel program to plot the curves. The peak in Figure 4 is about 17 points wide at FWHM. Visually, the 13-pt and 17-pt $S-G$ filters appear to smooth the curve and fit the data points the best. For the smaller filter sizes, the fitted curves are following the noise oscillations. Close examination of the inflection points of the various curves in Figure 4 show that they are very close to the same point. The inflection point is the place where the second derivative equals zero and Figure 3 shows the variation in the location of this point for the different filters used. Although the inflection points are relatively insensitive to the number of points in the S-G filter, as the number of points in the filter increases above that which would best fit the peak, the maximum value in the fitted curve is decreasing with the result that the FWHM value increases. However, and this is the main point of this article, if the appropriate sized filter is used, the FWHM value of the fitted peak is little affected by the filter.

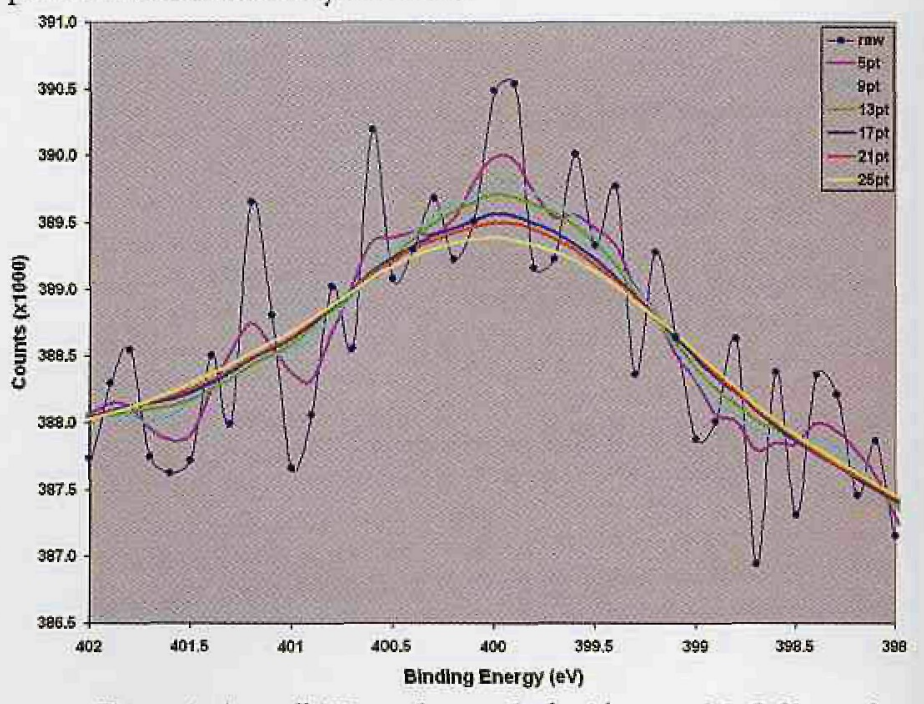

Figure 4 A small Nis peak smoothed with several $S-G$ filters of different sizes. The curve fitting the points is from the smoothing option in Microsoft" Excel Chart. 


\section{The Next Step...}

\section{Innovative Solutions for Nano-Technology}

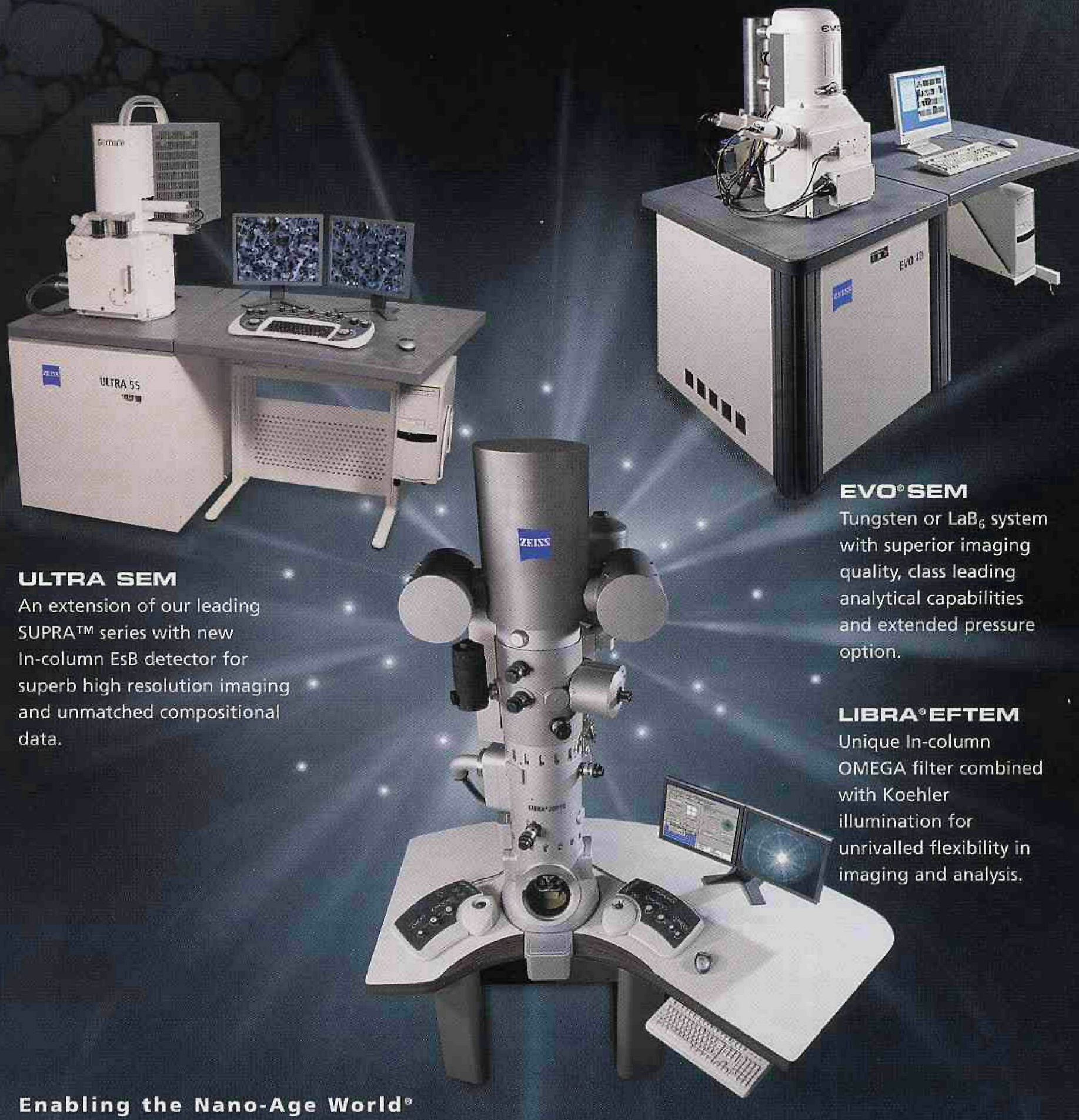

Carl Zeiss SMT Inc

One Zeiss Drive, Tharnwood

New York 10594

USA
Tel. $+1914 / 7477700$

Fax $+1914 / 6817443$

info-usa@smt.zeiss.com

www.smt.zeiss.com/nts 
Table 3: Savitsky-Golay First Derivative Coefficients for Quadratic Fit

\begin{tabular}{|c|c|c|c|c|c|c|c|c|c|c|c|c|}
\hline & $m$ & 2 & 3 & 4 & 5 & 6 & 7 & 8 & 9 & 10 & 11 & 12 \\
\hline$s$ & $2 m+1$ & 5 & 7 & 9 & 11 & 13 & 15 & 17 & 19 & 21 & 23 & 25 \\
\hline & & & & & & & & & & & & \\
\hline-12 & & & & & & & & & & & & 0.0034 \\
\hline-11 & & & & & & & & & & & 0.0043 & 0.0026 \\
\hline-10 & & & & & & & & & & 0.0056 & 0.0032 & 0.0018 \\
\hline-9 & & & & & & & & & 0.0075 & 0.0040 & 0.0021 & 0.0011 \\
\hline-8 & & & & & & & & 0.0103 & 0.0050 & 0.0024 & 0.0011 & 0.0004 \\
\hline-7 & & & & & & & 0.0147 & 0.0064 & 0.0028 & 0.0011 & 0.0003 & -0.0001 \\
\hline-6 & & & & & & 0.0220 & 0.0084 & 0.0031 & 0.0009 & -0.0001 & -0.0005 & -0.0006 \\
\hline-5 & & & & & 0.0350 & 0.0110 & 0.0031 & 0.0003 & -0.0007 & -0.0010 & -0.0011 & -0.0010 \\
\hline-4 & & & & 0.0606 & 0.0140 & 0.0020 & -0.0013 & -0.0021 & -0.0021 & -0.0018 & -0.0016 & -0.0013 \\
\hline-3 & & & 0.1190 & 0.0152 & -0.0023 & -0.0050 & -0.0047 & -0.0039 & -0.0031 & -0.0025 & -0.0020 & -0.0016 \\
\hline-2 & & 0.2857 & 0.0000 & -0.0173 & -0.0140 & -0.0100 & -0.0071 & -0.0052 & -0.0038 & -0.0029 & -0.0023 & -0.0018 \\
\hline-1 & & -0.1429 & -0.0714 & -0.0368 & -0.0210 & -0.0130 & -0.0086 & -0.0059 & -0.0043 & -0.0032 & -0.0024 & -0.0019 \\
\hline 0 & & -0.2857 & -0.0952 & -0.0433 & -0.0233 & -0.0140 & -0.0090 & -0.0062 & -0.0044 & -0.0033 & -0.0025 & -0.0019 \\
\hline 1 & & -0.1429 & -0.0714 & -0.0368 & -0.0210 & -0.0130 & -0.0086 & -0.0059 & -0.0043 & -0.0032 & -0.0024 & -0.0019 \\
\hline 2 & & 0.2857 & 0.0000 & -0.0173 & -0.0140 & -0.0100 & -0.0071 & -0.0052 & -0.0038 & -0.0029 & -0.0023 & -0.0018 \\
\hline 3 & & & 0.1190 & 0.0152 & -0.0023 & -0.0050 & -0.0047 & -0.0039 & -0.0031 & -0.0025 & -0.0020 & -0.0016 \\
\hline 4 & & & & 0.0606 & 0.0140 & 0.0020 & -0.0013 & -0.0021 & -0.0021 & -0.0018 & -0.0016 & -0.0013 \\
\hline 5 & & & & & 0.0350 & 0.0110 & 0.0031 & 0.0003 & -0.0007 & -0.0010 & -0.0011 & -0.0010 \\
\hline 6 & & & & & & 0.0220 & 0.0084 & 0.0031 & 0.0009 & -0.0001 & -0.0005 & -0.0006 \\
\hline 7 & & & & & & & 0.0147 & 0.0064 & 0.0028 & 0.0011 & 0.0003 & -0.0001 \\
\hline 8 & & & & & & & & 0.0103 & 0.0050 & 0.0024 & 0.0011 & 0.0004 \\
\hline 9 & & & & & & & & & 0.0075 & 0.0040 & 0.0021 & 0.0011 \\
\hline 10 & & & & & & & & & & 0.0056 & 0.0032 & 0.0018 \\
\hline 11 & & & & & & & & & & & 0.0043 & 0.0026 \\
\hline 12 & & & & & & & & & & & & 0.0034 \\
\hline
\end{tabular}

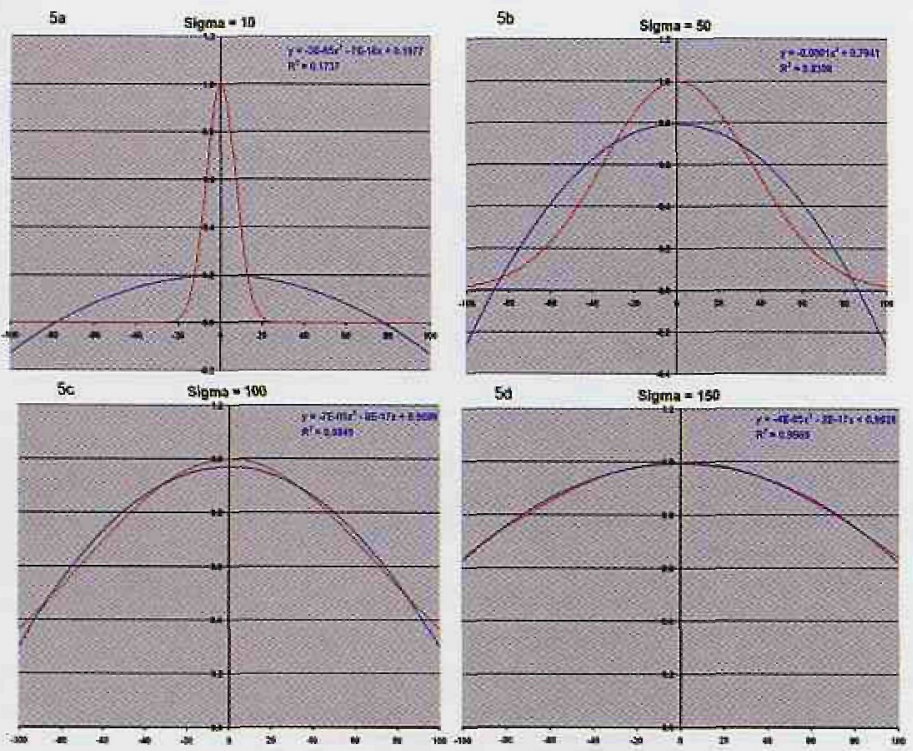

Figure 5 Four Gaussian curves with the indicated $\sigma$ values and quadratic fitting curves (trendlines) fitted between -100 and 100. As the Gaussian uses more of the range, the second-degree polynomial becomes a better fit as measured by the R2 term.

The effects that have been discussed here can also be illustrated nicely by using Microsoft ${ }^{\circ}$ Excel to model a Gaussian peak and to fit it with a polynomial trendline. In the examples in figure 5, Gaussian peaks were created using the expression,

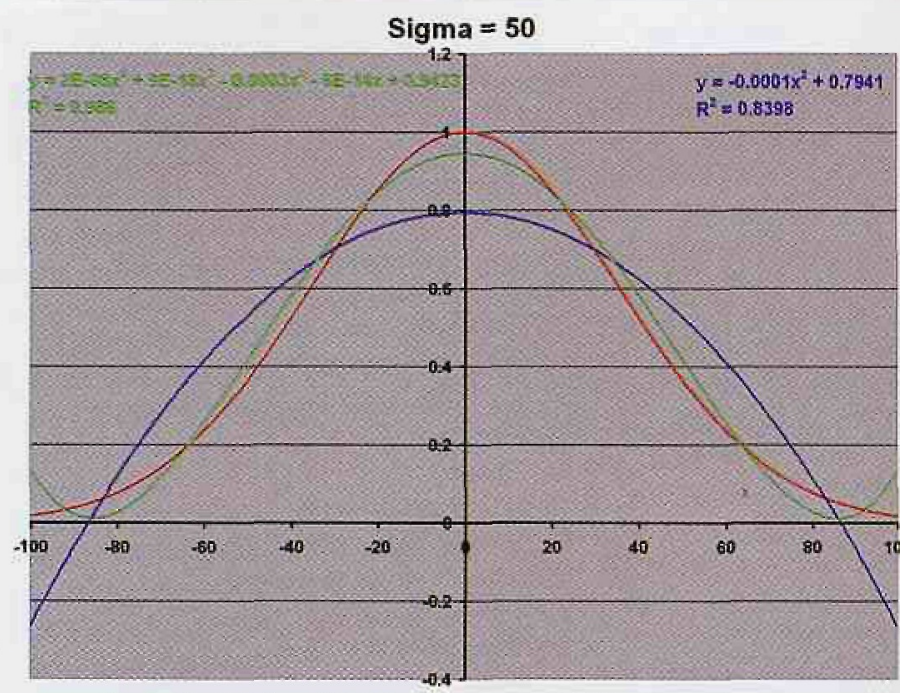

Figure 6 A 2nd and 4th degree polynomial fitted to a Gaussian peak with $\sigma=50$.

$$
Y=A \cdot \exp ^{\left(-x^{2} / \sigma^{2}\right)}
$$

with $A=1$, and -100 t $x \nmid 100$ in 0.05 increments. The value for $\sigma$ was changed in order to change the expanse of the curves over this range. As $\sigma$ increases, the fit of a quadratic trendline fitted over the range becomes a better fit, as measured from the $R^{2}$ value. In Figure 5, the values for $\sigma$ are 10, 50, 100, and 150 with corresponding $R^{2}$ values of $0.1737,0.8398,0.9849$, and 0.9969 , respectively; the latter being nearly a perfect fit. Excel could also be used to illustrate the effect of using a higher degree polynomial for a S-G smoothing filter. In Figure 6 , both a $2^{\text {nd }}$ and $4^{\text {th }}$ degree polynomial trendlines were fitted to the Gaussian curve having a $\sigma$ value of 50 . Clearly, the $4^{\text {th }}$ degree polynomial is shown to follow the curvature of the peak better, with an $R^{2}$ value of 0.986 compared to 0.8398 for the $2^{\text {nd }}$ degree polynomial.

In closing this article, I would also like to make a small point about inflection points and measuring the actual resolution of Gaussian shaped peaks. As was seen, the inflection points were very nearly the same when the different size S-G filters were used. If equation ( 1 ) is solved for $Y=1 / 2 A$, then the solution is $x=\sigma \cdot(\ln 1 / 2)^{1 / 2} \approx \pm 0.833 \sigma$. These are the two values of $x$ at the FWHM in terms of $\sigma$. If the second derivative of equation 1 is set to zero to find the inflection points, then they occur at $x= \pm$ $1 / 2 \sqrt{2} \cdot \sigma \approx \pm 0.707 \sigma$. Several S-G filters could be used to determine the inflection points of the curve, and then the above relationships could be used to determine a reliable value for the resolution of the peak.

\section{Endnotes}

${ }^{1}$ A. Savitsky and M. J. E. Golay, Anal. Chem. 36, pp. I627-39, (1964).

${ }^{2}$ J. Steiner, Y. Termonia, J. Deltour, Anal. Chem, 44, pp. 1906-9, (1972):

${ }^{3}$ H. H. Madden, Anal. Chem., 50, pp. 1383-6, (1976).

${ }^{4}$ The Top-Hat filter is described in most XEDS software package manuals. I used the technical reference manual for the Noran Vantage system. I also benefited from discussions with Fred Schamber and he was kind enough to send me several of his reprints on the topic.

${ }^{5}$ EELS-Plot is a Microsoft" Visual Basic 6,0 EELS and XEDS program that works with MSA formatted spectra in a Microsoft" Windows" platform. It is downloadable from the EMMPDL library at the following web site, http://www.amc. anl.gov/ANLSoftwarel ibrary/02-EMMPDL/EELS/EELSPlot/. 


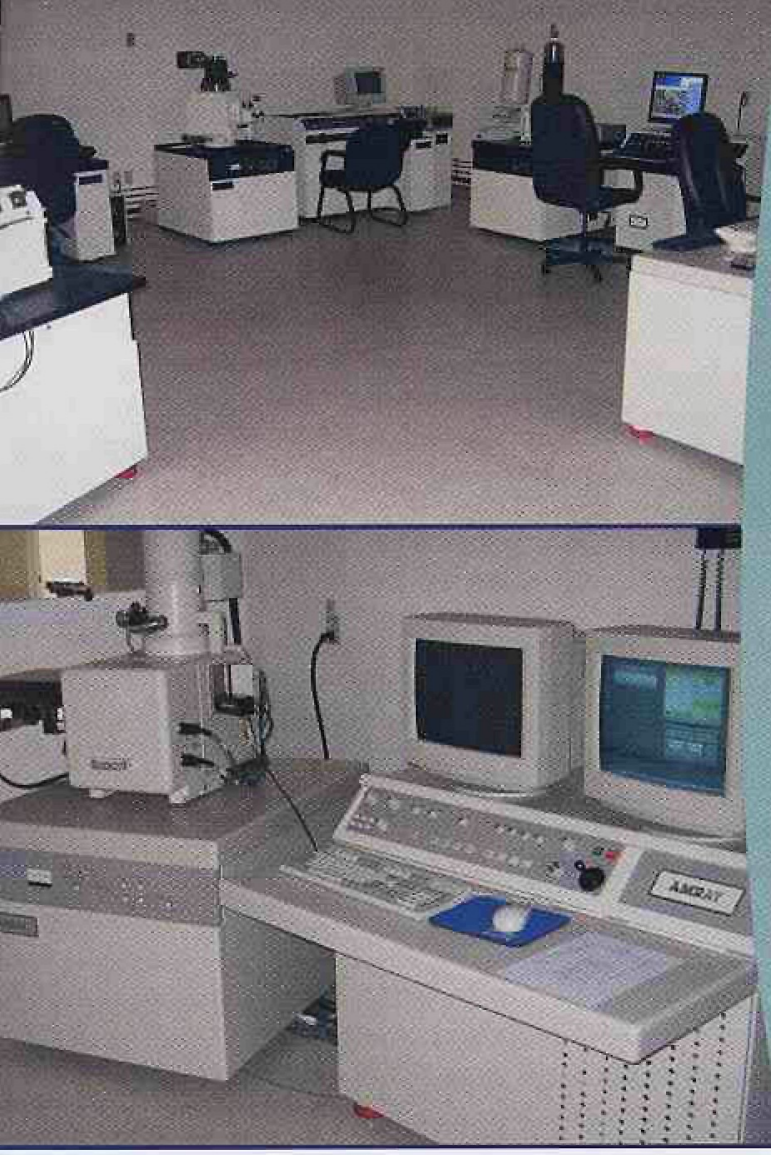

\section{Pre-Owned SEMs -} The Smart \& Easy Alternative to Buying New

Why buy a new Scanning Electron Microscope when SEMTech Solutions offers a smarter and easier solution?

We specialize in pre-owned, rebuilt and upgraded products, including:

- Field Emission SEMs

- Digital Imaging

- Computer and EDS Upgrades

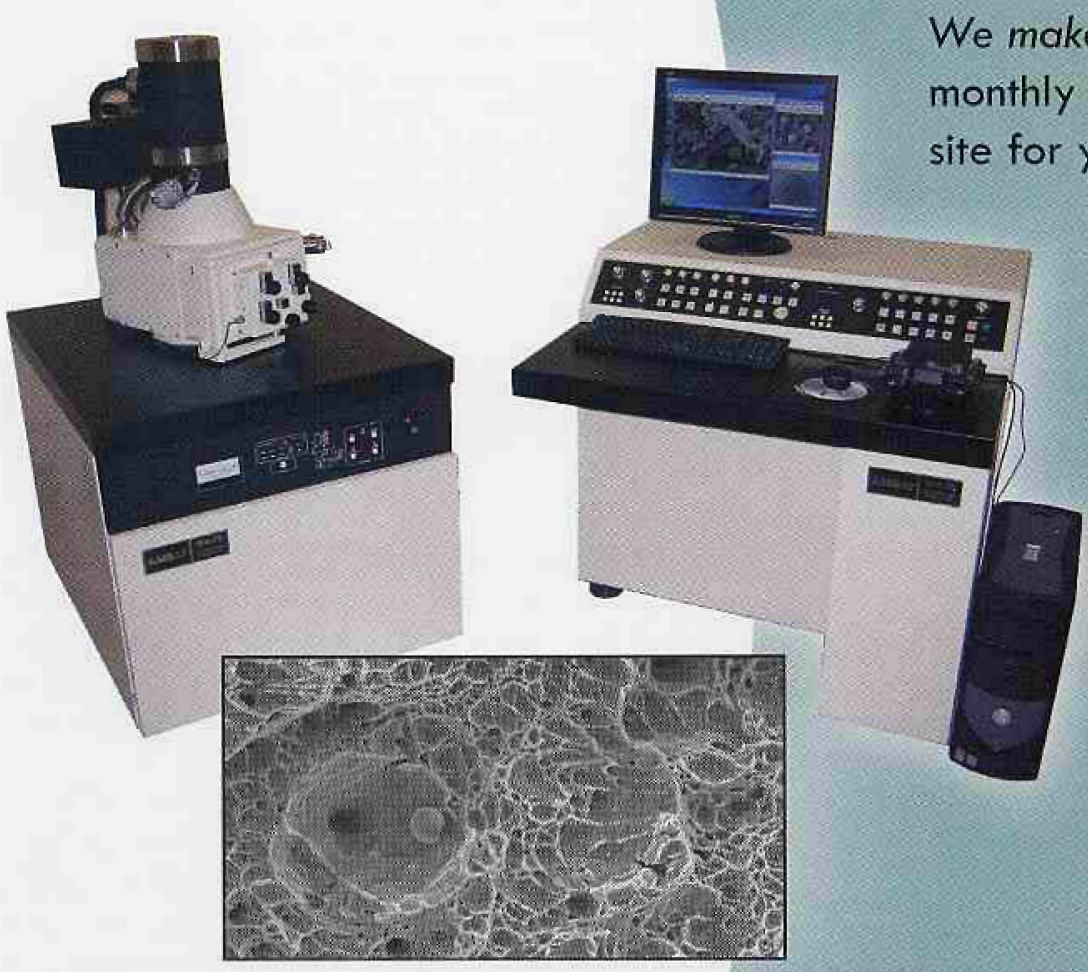

We make it easy by offering hands-on, in-person equipment demonstrations at our Massachusetts facility. Scheduling a demo is simple; just call us at $978 \cdot 663 \cdot 9822$ for an appointment.

Visit us at

www.semtechsolutions.com to get more information on all SEMTech Solutions products and services.

Smart. Easy.

- SEMTech Solutions, Inc.

SEMTech Solutions, Inc.

6 Executive Park Drive

North Billerica, MA 01862

$978 \cdot 663 \cdot 9822$

www.semtechsolutions.com 